\title{
Poesia: Ti diranno di non splendere
}

\section{Fernando Della Posta \\ Poeta, scrittore. Roma}

Ti diranno di non splendere

ricordare le ceneri

sparse sui fondali dei robivecchi

cercando gli operai che non sono.

fasci di muscoli iniettati di sangue

e martelli levati.

trovare toni di voce sbracati

lingue adatte ad affilare coltelli,

minacce e bestemmie

sull'ideale che fu.

santini in tasca come talismani.

viola sanguigna non colta dai più

lasciata al macero degli anni

nelle teche dei saloni sepolti.

le falci solerti non mietono più

nutrimento dalle pagine ingiallite 
e le rondini volano a pelo d'acqua

senza rischiare la vita. i ricci di mare

negli antipasti sontuosi borghesi

degli italiani, deflagrano chicchi di sale

sui mortai dei campi rom.

faccia da bimbo in bianco e nero pensoso

la tua canaglia forse non cambia?

crudele e sublime rischiare

un pianto supremo di madre

per una manciata d'ossa e piume.

forse una fiaba temeraria

e consolante, che non meraviglia più?

Ilaria partoriente di Lucca

metafora da figlio prodigio

sacrificato sull'altare più alto

della più spietata borghesia di rito.

Già condannato mentre denunciava:

libera Ilaria dall'Italia, il mondo!

Saturo nel grembo freddo

nucleo caldo sovrumano infecondo,

squassato dal porno/fascio perverso:

zavorra sul volto d'llaria

che dorme inerme e fatata.

risveglia l'anima del popolo, 
fiaccato dal vivere onesto!

- madre dimenticata e figlio protesto:

cosi che ogni filo infine passi

per la sua cruna dorata:

che si liberino gli occhi sereni

del volto soave d'llaria!

Che sia di verso osceno

di storia sbagliata

il nominarti a denti stretti

un punto di domanda.

L'obbrobriosa condanna

fabbricata per la bocca dei mentecatti

si muove in circolo e scolora

pochi istanti.

Più forte l'anima si riempie

se considera il meticoloso

esplosivo disegno dei versi,

la capillare descrizione dei censi,

l'ostinata dichiarazione di sé:

se deglutisce il groppo

senza perdonare l'imperdonabile

ché nulla c'è da perdonare:

"ti diranno di non splendere,

e tu splendi, invece!" 
Fernando Della Posta. Nato nel 1984 a Pontecorvo in provincia di Frosinone, è laureato in Scienze Statistiche, vive a Roma e lavora nel settore informatico. Tra i tanti riconoscimenti ottenuti in poesia nel 2011 è arrivato tra i finalisti al concorso di poesia "Ulteriora Mirari" nella sezione silloge poetica inedita; nel 2015 è risultato tra i finalisti del concorso letterario "Sistemi d'Attrazione", legato al festival "Bologna in lettere 2015", nella sezione dedicata a Pier Paolo Pasolini; nel 2016 vince il concorso "Stratificazioni: Arte-fatti Contemporanei" legato al festival letterario di Bologna in Lettere 2016 nella sezione B poesia inedita a tema libero e ottiene una menzione al XXX premio Montano per la silloge inedita. Nel 2017 vince il Premio Nazionale Poetika nella sezione silloge inedita. Nel 2018 si classifica secondo nella sezione inediti di poesia al Premio "Andrea Torresano", ottiene una segnalazione al premio Lorenzo Montano per la silloge inedita e vince il Premio Letterario Zeno nella sezione poesia. Nel 2019 ottiene piazzamenti da finalista per la raccolta inedita ai concorsi: "Paul Celan" e "Pietro Carrera". Sempre per la raccolta inedita ottiene la segnalazione al Lorenzo Montano. Sempre nello stesso anno, inoltre, ottiene il secondo posto nella poesia inedita e la menzione di merito per il libro edito "Voltacielo" al premio Chiaramonte Gulfi. Numerose sono le sue recensioni e le sue sillogi reperibili su diversi blog letterari come Neobar, di cui è redattore, Words Social Forum, Viadellebelledonne, Poetarum Silva, L'EstroVerso e II Giardino dei Poeti. Nel 2011 ha pubblicato la raccolta di poesie "L'anno, la notte, il viaggio" per Edizioni Progetto Cultura e, sempre in poesia, nel 2015 "Gli aloni del vapore d'Inverno" per Divinafollia Edizioni, nel 2017 "Cronache dall'Armistizio" per Onirica Edizioni, nel 2018 "Gli anelli di Saturno" per Ensemble Edizioni e nel 2019 "Voltacielo" per Oèdipus Edizioni. 